\title{
Effects of in-water and dryland warm-ups on 50- meter freestyle performance in child swimmer
}

\author{
Fatih Kaya $^{1 \mathrm{a}}$, Mustafa Said Erzeybek ${ }^{2}$, Bilal Biçer ${ }^{3}$, and Tuncay Meral ${ }^{4}$ \\ ${ }^{1}$ Faculty of Education, Department of Physical Education and Sports, Erzincan University, Turkey \\ ${ }^{2}$ School of Physical Education and Sports, Dumlupınar University, Turkey \\ ${ }^{3}$ School of Physical Education and Sports, Mustafa Kemal University, Turkey \\ ${ }^{4}$ Erzincan Municipality Sports Club, Erzincan, Turkey
}

\begin{abstract}
In this study, effectiveness of 3 warm-up (WU) modes on $50 \mathrm{~m}$ free style on child swimmers has been evaluated. In repeated-measures counterbalanced design, 10 male swimmers of a local sports club (10-12 ages) have tried out $50 \mathrm{~m}$ freestyle after each WU on different days. Each WU took 15 minutes and the intensity of WU has been checked over by Borg's rating of perceived exertion (RPE) 15-point scale. a) no warm-up (NWU): the swimmers sat or laid down for 15 minutes, b) dryland warmup (DWU): continuous rope-jumping at RPE 12 for 5 minutes, 2 sets $10 \times 15 \mathrm{~s}$ calisthenics with $15 \mathrm{~s}$ breathing spaces and continuous ropejumping at RPE 14 for 5 minutes, c) in-water warm up (SWU): continuous freestyle swimming at RPE 12 for 5 minutes, freestyle swimming with $8 \times 15 \mathrm{~m}$ maximum performance with $30 \mathrm{~s}$ breathing spaces and continuous freestyle swimming at RPE 14 for 5 minutes. RPE, stroke count and heart rate (HR) have been evaluated together with the best time in swimming performance. Compared with NWU swimming time post DWU was faster, however the fastest swimming time was achieved post SWU. After NWU swimming RPE was significantly higher. Significant difference could not be found in terms of HR and stroke count. Consequently, SWU is better for swimming performance and DWU can be used when swimming pool is not available.
\end{abstract}

Keywords: 50-m freestyle, warm-up, child swimmer

\section{Introduction}

Muscle and inner body temperature are needed to be increased before competition at sportive performance. Increase at body temperature prepares the body before serious competitions. A 1-2 degrees increase on body temperature is important in terms of mechanic and bioenergic efficiency and for this significant temperature increase, at $\% 5-70$ of maximum heart rate minimum 10-15 minutes exercise is known to be needed [7]. By

a Corresponding author: fkaya@erzincan.edu.tr 
means of these thermal effects of warm up (WU), disintegration of oxygen from hemoglobin and myoglobin increases [7, 15], energy generation increases through accelerating enzyme kinetics and substrate use [2, 16], viscosity of running muscles decreases $[15,16]$, speed of neural transmission and neural receptor sensitivity increases $[8$, 15], blood flow on active skeletal muscles increases [10, 11, 16] and lipid metabolism increases $[2,9]$.

Swimming is a branch of sports requiring muscular force and endurance and the winner is determined with only seconds even with split seconds. A good swimming performance is affected not only from exercise, genetic or physical fitness but also from WU which is also agreed as a key element on sportive performance [5]. Swimmers resort to increase their performances via WU just before competition. Even though it is possible to increase body temperature (active and passive) in different activities, in-water WU will probably be much more effective for swimming competition. Specific WU has been indicated to be much more superior than warm ups that are not specific to the branch. This situation enables the competitor's special motor units to work much more efficiently as well as it provides practice effect $[14,15]$. By this means, the swimmers get used to dive blocks, strips, return flags and wall surfaces for return. Besides this, dryland WU exercises to warm up, when swimming pool is not available, will make the swimmer much more prepared relatively to no warm up due to the increase at body temperature.

Even though there are studies researching different WU protocols' effects on swimming performance $[3,14,17]$ this situation have not been researched enough on child swimmers at competition level.

In this study, activity of three WU modes - in-water warm-up (SWU), dryland warm-up (DWU), and no warm-up (NWU) - on 50 meter (50m) free style swimming performance on child swimmers has been evaluated.

\section{Method}

In repeated-measures counterbalanced design, 10 child swimmers of a local sports club had $50 \mathrm{~m}$ freestyle trials after each 3 different WU modes on different days. The reason why $50 \mathrm{~m}$ swimming performance was chosen, it is the shortest event in a swimming competition. Because, at longer swimming events, the swimmers perform multi-returns during competition and longer swimming durations and longer swimming distances can decrease the effect of WU. In addition to 50m swimming performance, effects of WU have been evaluated on variables such as stroke count, heart rates (HR) and rating of perceived exertion (RPE).

\subsection{Subject}

10 male athletes between the ages 10-12 having at least 2 years swimming experience and having swimming experiences at official competitions participated in this study voluntarily. The participants and each of their legal representatives read "informed consent forms" and signed them. During the study, the participants were at season-end period and had 3 workouts a week.

In order to provide coherent conditions at trials, the subjects were requested to 1) avoid physical activities every test morning, 2) come to the pool by a vehicle rather than walking or cycling, 3) continue usual diet and sleeping patterns and 4) perform the highest performance at each test.

Besides, they were requested to avoid caffeine and physical activities (not related to swimming practices) at least 24 hours before each session, to avoid heavy food at least 3 hours before each session and not to consume energy drink. 
The participants having one of the following factors were excluded from the study: (a) if had a shoulder mutilation in the last 6 months; (b) if had a cardiovascular or respirator problem; (c) if had other chronic diseases restricting to participate in exercises; (d) if had a physical injury or surgical operation which makes the participation to exercise risky.

In order to minimize the errors that can arise in experiment period, one of the researchers got involved in the workouts of the children as their trainer before tests for one week. In this period, the children were comprehended the motions that will be performed in WU protocols and they were provided to be able to comment RPE cards. Also, they were taught to find and count pulse from neck or wrist. At this phase, the children were not informed about the research in detail to prevent practice effects arise at experiment period.

When it came to experiment period, the study design and procedure were explained to the children in detail before test sessions. By showing RPE charts, the RPE numbers were reminded verbally and visually.

Ages, heights and weights of the participants were recorded. Simbo SBS4432 bascule was used for body weight and 1/100s scaled EPSAN electronic chronometer (Multistar EP69052) for swimming times.

\subsection{Experimental procedure}

\subsubsection{Warm-up modes}

The WU protocols, which were previously used by Romney and Nethery [14] were used by remodifying. At counterbalanced design, each subject performed a $50 \mathrm{~m}$ freestyle trial at maximum effort after each WU protocol. Between each trial period, 48 hours relaxation was given. All testing was done at 8 A.M.

Swimming performance started 3 minutes after the WU completed. 15 minutes WU protocols are as following: 1) No warm-up (NWU): the subjects sat or laid down on an exercise mat for 15 minutes. 2) Dryland warm-up (DWU) : a) RPE= at 12 (fairly lightsomewhat hard) continuous rope-jumping for 5 minutes; b) at 5 minutes periods pushups, sit-ups, lunge jump, back hyperextensions, surgical tubing butterfly pulls (2 sets, each exercise $15 \mathrm{~s}, 15 \mathrm{~s}$ rest in between); c) $\mathrm{RPE}=$ at 14 (somewhat hard) continuous ropejumping for 5 minutes; 3) In-water warm-up (SWU): a) RPE=at 12, 5 minutes continuous freestyle swimming b) $8 \times 15 \mathrm{~m}$ freestyle sprints for 5 minutes (each at $30 \mathrm{~s}$ rest intervals and each run at $50 \mathrm{~m}$ competition speed); and c) $\mathrm{RPE}=$ at 14 , continuous freestyle swimming for 5 minutes.

In order to determine the intensity of the WU, RPE assessment was carried out at first and last $5 \mathrm{~min}$. sessions of the each WU activity. The intensity of the WU was prescribed with RPE relatively to heart rate and monitored because of the reasons like; highconductivity of the water effects the signals coming from heart rate monitor, maximum heart rates are 10-13 pulses lower in a minute relatively to exercises like rope jump, maximum heart rates often change by 20 pulses a minute on age mates [12].

\subsubsection{0m maximal performance trial}

The subjects were allowed to use bonnets and goggles, to sit, to stand, to walk, or to talk as might be done preceding a competitive event. Any motions including warm-up were not allowed behind block. The same lane was assigned to each subject at trials.

Standard starting procedure was used for swimmers climbing dive block: the researcher's voice command (take your mark) after electronic bip sound time was started. The time was stopped when the participant touched swimming wall. During swimming, 2 
persons followed each swimmer and 2 chronometers were used for their swimming times. Average of the two chronometers was recorded as the best swimming time.

\subsubsection{Rating of perceived exertion}

The participants were requested to scale their warm-ups and $50 \mathrm{~m}$ swimming performances by using Borg's 15 point scale [6]. RPE was determined after warm-up and after $50 \mathrm{~m}$ swimming performance.

\subsubsection{Heart rate}

Heart rate was measured by manual palpation method at the wrist or neck. All the participants were familiar with this procedure because they used this method in regular swimming training. The heart rate was measured 30 seconds before the swim trial and immediately after the $50 \mathrm{~m}$ maximal performance trial. The participants counted their own HR for 15 seconds. Heart rate was reported as beats per minute.

\subsubsection{Stroke count}

During the 50m maximal performance trial, strokes were counted. However, to minimize error, stroke count was counted in cycles. One cycle was equal to 2 strokes. The cycle started when the first arm of the participant entered the water and it ended when it recovered.

Outdoor environment temperature: $24 \mathrm{C}^{0}$

Water Temperature: $26.5 \mathrm{C}^{0}$

$\mathrm{Ph}: 7.4$ (ideally 7.2 )

Moisture: $\% 20$ (rises till $\% 50$, adjusted to $20-25$ by air-conditioners)

Chlorine: 1.4 (between 1-1.5)

Relative chlorine: 0.02 (between 0.02-0.04)

\subsubsection{Statistical analysis}

One-way repeated-measures ANOVA was used to analyze the difference in $50 \mathrm{~m}$ freestyle time trial performance, stroke count, RPE, and HR between the three different WU protocols. Significant was accepted at an $\mathrm{p}<0.05$ level. When a significant $\mathrm{F}$ ratio was obtained, a post hoc multiple comparison test was used to determine the location of the significant differences. All data reported as the mean $\pm \mathrm{SE}$.

\section{Findings}

Basic characteristics of the subjects are demonstrated at Table 1.

Table 1. Participants' age, weight, swimming experience, best $50 \mathrm{~m}$ time, and weekly training distance. Mean (SE)

\begin{tabular}{lc}
\hline Characteristic N=10 & \\
\hline Age $(\mathrm{yrs})$ & $11.00(0.21)$ \\
Height $(\mathrm{cm})$ & $146.20(2.59)$ \\
Weight $(\mathrm{kg})$ & $37.75(2.28)$ \\
HR rest $\left(\mathrm{b} . \mathrm{min}^{-1}\right)$ & $68.80(2.52)$ \\
Yrs. Swimming & $2.50(0.22)$ \\
Weekly training $(\mathrm{km})$ & $10.70(1.05)$ \\
Best 50m time $(\mathrm{s})$ & $41.38(1.66)$ \\
\hline
\end{tabular}


Table 2. Effects of three different warm-ups on variables. Mean (SE)

\begin{tabular}{lccc}
\hline & NWU & DWU & SWU \\
\hline 50m time (s) & $42.78(1.97) \mathrm{a}$ & $41.67(1.66) \mathrm{b}$ & $40.47(1.62) \mathrm{bc}$ \\
HR pre 50 $\left(\mathrm{b} \cdot \mathrm{min}^{-1}\right)$ & $82.40(2.33) \mathrm{a}$ & $113.60(5.85) \mathrm{b}$ & $110.40(6.65) \mathrm{bc}$ \\
HR post 50 $\left(\mathrm{b} \cdot \mathrm{min}^{-1}\right)$ & $134.80(8.83)$ & $140.00(9.02)$ & $133.60(7.30)$ \\
WU RPE & $7.20(0.20) \mathrm{a}$ & $10.80(0.77) \mathrm{b}$ & $12.00(0.49) \mathrm{bc}$ \\
50m RPE & $14.90(0.35) \mathrm{a}$ & $14.20(0.42) \mathrm{b}$ & $13.90(0.31) \mathrm{bc}$ \\
Stroke count & $31.25(0.92)$ & $31.55(1.16)$ & $31.50(0.89)$ \\
\hline \multicolumn{2}{c}{ Means sharing the same subscript are not significantly different from each other }
\end{tabular}

Findings obtained show that there is significant difference among three WU protocols for $50 \mathrm{~m}$ swimming performance, $\mathrm{F}(2,18)=8.547, \mathrm{p}=0.00$ (Table 2). When post hoc comparisons were compared with NWU (42.78s), it revealed that average 50m swimming time $(41.67 \mathrm{~s})$ after DWU is significantly better $(\mathrm{p}=0.03)$, however the best swimming time was revealed to be achieved after SWU (40.47s), ( $p=0.01$, Figure 1).

Furthermore, RPE scores after WU and after swimming were significantly different [respectively $\mathrm{F}(2,18)=24.850, \mathrm{p}=0.00 ; \mathrm{F}(2,18)=5.427, \mathrm{p}=0.01$, Table 2]. Post hoc comparisons showed that after warm-up RPE scores are higher at DWU $(10.80=60 \%$ near to effort) and at SWU (12.00=65\% effort), (respectively $\mathrm{p}=0.01 ; \mathrm{p}=0.00)$. In addition to this, RPE scores after NWU $(14.90=80 \%$ near to effort $)$ are significantly higher respectively to both WU protocols (respectively $\mathrm{p}=0.03 ; \mathrm{p}=0.03$ ).

There was significant difference at $\mathrm{HR}$ before swimming, $\mathrm{F}(2,18)=11.258, \mathrm{p}=0.00$. Compared with NWU, while being higher at DWU $(p=0.01)$ and SWU $(p=0.02)$, significant difference could not be found for after swimming HR and stroke counts [respectively $\mathrm{F}(2,18)=0.717, \mathrm{p}=0.50 ; \mathrm{F}(2,18)=0.199, \mathrm{p}=0.82$, Table 2].

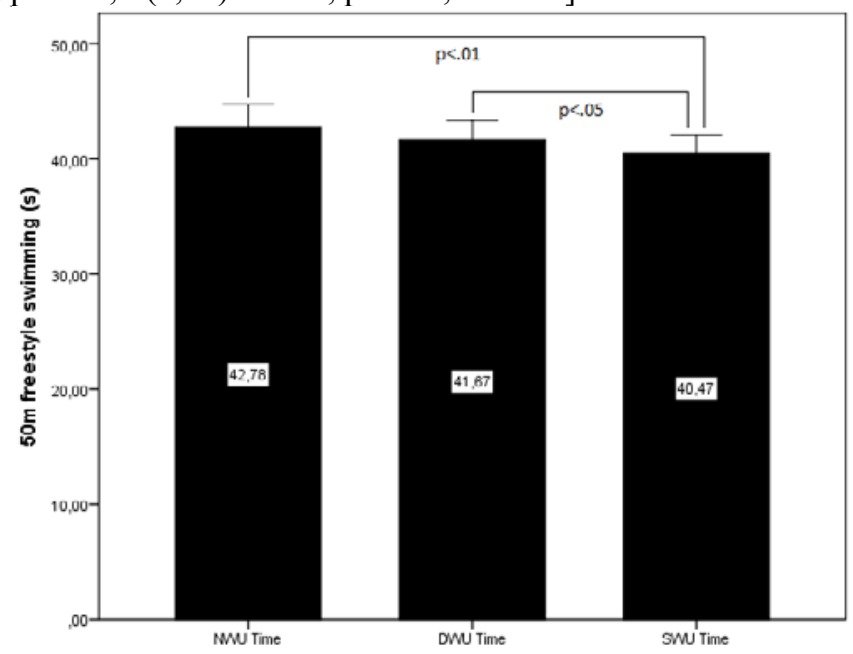

Fig. 1. Mean performance times for $50 \mathrm{~m}$ freestyle following no warm-up, dryland warm up, and inwater warm up (+/-1 SE).

\section{Discussion}

The outcomes obtained in this study show that the best $50 \mathrm{~m}$ swimming time has been achieved after in-water WU. Besides this, when compared with NWU, after DWU 50m swimming performance showed significantly better result. These findings have showed the activity of WU's ergogenic value both theoretically and practically again. With WU; body temperature, blood flow, respiratory rate, heart rate and flexibility of the needed muscles 
increase [10]. Moderate and not lesser than 5 minutes WU has also been reported to increase muscle and body temperature $[1,4,18]$. Intensity and duration of the in-water and dryland WU protocols used in this study mean to be sufficient to increase muscle and body temperature (despite the body temperature wasn't measured directly). As we have already stated, maximum \%65-70 MUSCLE and at least 10-15 minutes exercise are necessary in order to increase significant body temperature [7]

Similar results were achieved in Romney et al. [14] study, we used their WU protocols by remodifying, and they explained this with a number of physiological alterations: decrease of tissue resistance with the systemic increase and increase in tissue heat, facilitation in transportation and distribution of oxygen, increase of usability and energy generation of substrate, getting ready of cardiorespiratory system. However in the abovementioned study, increase in swimming performance after dryland WU was accepted significant by researchers although it wasn't and they stated dryland WU can be sufficient too for physiological preparation when swimming pool is not available. A significant point here is; the participants were in average 20 age group and evaluated swimming distance is 100 yard $(91.44 \mathrm{~m})$.

The fact that in-water WU is much more effective can be explained with a few reasons. First of all, in-water WU protocol is well-designed to increase body temperature. Secondly, in-water WU revealed the practice effect of specific WU. Environmental adaptation and neuromuscular facilitation are stated to positively effected by specific to skill WU [10]. By this means, the athletes get used to dive blocks, strips, return flags and wall surfaces during returns. Also in this study, the participants regularly practiced in the swimming pool in which the tests were carried out and they were accustomed to environmental conditions.

Besides, in limited availability of studies that research swimming performance by using different WU protocols, mostly WU activities based upon short/long distance or low/high intensity swimming have been investigated and the outcomes in these studies have been found contradictory to the evidences on usefulness of WU $[5,10,13]$. Reasons like sample structures and wide age variable have been demonstrated as the basis of contradiction. Moreover, low intensity WU have been stated not to increase body temperature sufficiently and high intensity WU to cause fatigue [3].

Another finding achieved is RPE after NWU is significantly higher. This finding shows that the participants much more slog when they perform swimming performance without WU. 14.90 RPE score means the participants make about $\% 80$ effort. This condition is normal and it is accepted as an ordinary condition likely to be encountered when the body is not ready for performance. Thusly RPE scores are slightly lower in both WU protocols and the scores show that they feel the effort almost \% 70-75.

An interesting finding achieved from this study that there wasn't a difference between swimming protocols in terms of HR. Eventhough the participants felt the swimming trial after NWU compelling in terms of RPE, there wasn't a difference in terms of HR. This finding is contradictory with the previous study results. In previous studies, rising HR results were achieved when after swimming performance compared with no WU. Bishop [4] stated that a rise can happen at HR based upon after WU muscle and core temperature and rising $\mathrm{VO} 2$ [4]. In addition to this, after low and high intensity swimming WU protocols, high HR and high VO2 were stated [13]. However, when after swimming RPE scores analyzed, RPE score felt at NWU was close to \%80 effort while the difficulty level felt after two WU protocols was about \%70/75 effort. Although RPE scores differentiate significantly after swimming, difficulty level felt indeed is almost similar at every three protocols. From this point, the fact that rising HR scores are similar, can be taken normally.

Similarly, there wasn't a significant difference at stroke counts between WU protocols. This finding promotes the study results by Balilionis et al. [3] who didn't find difference at stroke counts between $50 \mathrm{~m}$ and 200-m swimming performances [3-18]. In this study, it's 
seen that the swimmers swim the same distance with similar stroke counts. In addition to this, it is possible to achieve difference at trials in which swimming distance is longer.

Consequently; in water warm-up has come up with a better result at achieving the best $50 \mathrm{~m}$ freestyle swimming performance. On the other hand, it can be said that dryland warmups can be used for physiological preparation when swimming pool is not available.

\section{References}

1. Arnett, MG. Effects of prolonged and reduced warm-ups on diurnal variation in body temperature and swim performance. J Strength Cond Res 16, 256-261, (2002).

2. Åstrand, P.O., and K. Rodahl. Textbook of work physiology: New York: McGraw-Hill, (1986).

3. Balilionis, G., Nepocatych, S., Ellis, C. M., Richardson, M. T., Neggers, Y. H., \& Bishop, P. A. Effects of different types of warm-up on swimming performance, reaction time, and dive distance. J Strength Cond Res, 26(12), 3297-3303, (2012).

4. Bishop, D. Warm-up II. Performance changes following active warm-up and how to structure the warm up. Sports Med 33,483-498, (2003).

5. Bobo, M. The effect of selected types of warm-up on swimming performance. Int Sports J., 3, 37-43, (1999).

6. Borg, G. Perceived exertion: A note bon 'history' and methods. Med Sci Sports 5, 90-93, (1972).

7. DeVries, H.A. Physiology of exercise for physical education and athletics, Wm.C.Brown Company, (1974).

8. Franks, D. Physical warm-up. In: Ergogenic Aids in Sport. Melvin H. Williams (Ed.), Human Kinetics, Champaign, 340-375, (1980.),

9. Hetzler, Ronald K., et al. "Effect of warm-up on plasma free fatty acid responses and substrate utilization during submaximal exercise." Research Quarterly for Exercise and Sport 57(3), 223-228, (1986).

10. King, A. The relative effects of various warm-up procedures on 50 meter times of trained, competitive swimmers. J Phys Educ 7, 22-24, (1979).

11. Martin, BRUCE J., et al. "Effect of warm-up on metabolic responses to strenuous exercise." Medicine and science in sports 7(2), 146-149, (1974).

12. McArdle, W. D., Katch, F. I., \& Katch, V. L. Exercise physiology: nutrition, energy, and human performance. Lippincott Williams \& Wilkins, (2010).

13. Mitchell, JB and Huston, JS. The effect of high- and low-intensity warm-up on the physiological responses to a standardized swim and tethered swimming performance. J Sports Sci 11, 159-165, (1993).

14. Romney, Neil Conrad, and V. M. Nethery. "The effects of swimming and dryland warm-ups on 100-yard freestyle performance in collegiate swimmers." J Swim Res 9: 5-9, (1993).

15. Shellock, F.G. Physiological benefits of warm-up, Phys. And Sports Med. Oct., 135139, (1983).

16. Watson, A. W. S. Physical fitness and athletic performance. A guide for students, Athletes, and Coaches. London: Longman, (1983).

17. Wilson, E. E., McKeever, T. M., Lobb, C., Sherriff, T., Gupta, L., Hearson, G., \& Shaw, D. E. Respiratory muscle specific warm-up and elite swimming performance. $\mathrm{Br}$ J Sports Med, bjsports, (2013).

18. Zochowski, T, Johnson, E, and Sleivert, GG. Effects of varying post warm-up recovery time on 200-m time-trial swim performance. Int J Sports Physiol Perform 2, 201-211, (2007). 